\title{
A post-processing method to simulate the generalized RF sheath boundary condition
}

\author{
James R. Myra ${ }^{1, *}$ and Haruhiko Kohno ${ }^{2}$ \\ ${ }^{1}$ Lodestar Research Corporation, Boulder, Colorado, 80301, USA \\ ${ }^{2}$ Kyushu Institute of Technology, Iizuka, Fukuoka, 820-8502, Japan
}

\begin{abstract}
For applications of ICRF power in fusion devices, control of RF sheath interactions is of great importance. A sheath boundary condition (SBC) was previously developed to provide an effective surface impedance for the interaction of the RF sheath with the waves. The SBC enables the surface power flux and rectified potential energy available for sputtering to be calculated. For legacy codes which cannot easily implement the SBC, or to speed convergence in codes which do implement it, we consider here an approximate method to simulate SBCs by post-processing results obtained using other, e.g. conducting wall, boundary conditions. The basic approximation is that the modifications resulting from the generalized SBC are driven by a fixed incoming wave which could be either a fast wave or a slow wave. The method is illustrated in slab geometry and compared with exact numerical solutions; it is shown to work very well.
\end{abstract}

\section{Introduction}

For applications of ion cyclotron range of frequencies (ICRF) power in fusion devices, control of RF specific interactions with the scrape-off layer plasma and material surfaces is of great importance. Near these surfaces, sheaths on the scale of the Debye length form. For typical parameters of interest, i.e. RF sheath voltages much larger than the local temperature, these sheaths are strongly dominated by the presence of RF waves. The sheaths impact the wave fields and the local wave fields impact the sheaths. The rectification of rf voltages across the sheath is believed to contribute to power losses [1] and enhanced sputtering, the latter motivating the investigation of new antenna designs $[2,3]$.

Because of the scale separation between the sheath and global wave physics, and strong sheath nonlinearities, it is not practical to directly simulate RF sheaths in global ICRF waves codes. Instead, sheath boundary conditions (SBCs) and related methods have been developed [4 - 7] and implemented in global codes [8]. A recent general formulation [9] of the SBC provides an effective surface impedance for the interaction of the RF sheath with the waves. Furthermore, it enables quantities of interest for material interactions, such as the surface power flux and rectified potential energy available for sputtering, to be calculated.

Unfortunately, many legacy ICRF codes do not implement the SBC. For them it would be desirable to have an approximate method to simulate sheath boundary conditions by post-processing results obtained using other, e.g. conducting wall, boundary conditions.
This paper describes such a method, expected to be useful when the conducting wall code can accommodate boundaries which are not restricted to coincide with a flux surface. Furthermore, in codes with an SBC, iteration is required for a converged self-consistent solution since the SBC is nonlinearly dependent on the RF wave amplitude. The method may also be useful in speeding up convergence in these cases.

The basic approximation enabling the method is that the modifications resulting from the generalized SBC are driven by a fixed incoming wave which could be either a fast wave or a slow wave. Code data is first postprocessed to obtain the amplitude of the incoming waves. Then holding this amplitude fixed, the wave equations are solved again, approximately, in the vicinity of the wall sheath, using the SBC. The resulting solution contains modified RF fields in the vicinity of the wall as well as a modification of any reflected (outgoing) waves.

A general description of the method and algorithm is presented in Sec. 2. In Sec. 3 the post-processing method is applied to a sample problem in slab geometry, and the results are compared with a numerical solution using the rfSOL code $[8,10]$, a finite element RF wave code which now implements the full generalized SBC. Finally a discussion and conclusions are given in Sec. 4

\section{Description of the method}

The generalized sheath BC may be written in the form.

$$
\mathbf{E}_{\mathrm{t}}=\nabla_{\mathrm{t}}\left(\mathrm{J}_{\mathrm{n}} \mathrm{z}_{\mathrm{sh}}\right)
$$

\footnotetext{
Corresponding author: jirmyra@,lodestar.com
} 
where $J_{n}$ is the rf current normal to the wall, $\mathbf{E}_{t}$ is the projection of $\mathbf{E}$ tangential to the wall surface, $\nabla_{t}$ is the tangential gradient operator, and $z_{\text {sh }}$ is the sheath impedance. The rf sheath voltage is

$$
\Phi_{\mathrm{sh}}=-\mathrm{J}_{\mathrm{n}} \mathrm{Z}_{\mathrm{sh}}
$$

and the sheath BC simply matches the $\mathbf{E}_{\mathrm{t}}$ of the wave at the entrance to the sheath to that of the sheath itself. Inside the sheath $\Phi$ hence $\mathbf{E}_{\mathrm{t}}$ decays to zero on the surface of the conducting wall.

Although the method is quite general, for the sake of clarity we will describe the method for the case where the original code result was obtained using a conducting wall $\mathrm{BC}, \mathbf{E}_{\mathrm{t}}=0$. This corresponds to an original solution using $z_{\text {sh }}=0$ in Eq. (1). For our post-processing method, the data required from the original code result consists of $\mathrm{J}_{\mathrm{n}}$ and the tangential RF magnetic field $\mathbf{B}_{\mathrm{t}}$ for all points on the boundary.

In the vicinity of the wall four scalar equations describe the four possible modes in a cold plasma, two each describing different propagation directions for the fast wave (FW) and slow wave (SW). We will label these modes as $\mathrm{m}=1,2,3,4$. For each patch of wall region, taking the local conductivity tensor to be constant in this near-wall region, using the local eikonal approximation, and choosing a local coordinate system with the $\mathrm{x}$-direction normal to the wall, we have

$$
\begin{gathered}
\sum_{\mathrm{m}} \mathbf{e}_{\mathrm{x}} \cdot \overline{\bar{\sigma}} \cdot \mathbf{E}^{(\mathrm{m})}=\mathrm{J}_{\mathrm{x}} \\
\sum_{\mathrm{m}} \mathrm{E}_{\mathrm{y}}^{(\mathrm{m})}=\mathrm{i} \mathrm{k}_{\mathrm{y}} \mathrm{J}_{\mathrm{x}} \mathrm{z}_{\mathrm{sh}} \\
\sum_{\mathrm{m}} \mathrm{E}_{\mathrm{z}}^{(\mathrm{m})}=\mathrm{ik}_{\mathrm{z}} \mathrm{J}_{\mathrm{x}} \mathrm{z}_{\mathrm{sh}} \\
\sum_{\mathrm{m}}\left[\mathrm{n}_{\mathrm{z}} \mathrm{E}_{\mathrm{x}}^{(\mathrm{m})}-\mathrm{n}_{\mathrm{x}} \mathrm{E}_{\mathrm{z}}^{(\mathrm{m})}\right]=\mathrm{B}_{\mathrm{y}}
\end{gathered}
$$

Eq. (3) is the definition of the normal current in terms of the conductivity tensor while Eqs. (4) and (5) are the two components of the SBC. Eq. (6) is one component of the Maxwell equation $\mathbf{n} \times \mathbf{E}=\mathbf{B}$ where $\mathbf{n}=$ $\mathbf{k c} / \omega$. Instead of $\mathrm{B}_{\mathrm{y}}$ the equation for $\mathrm{B}_{\mathrm{z}}$ could be employed. ( $\mathrm{B}_{\mathrm{y}}, \mathrm{B}_{\mathrm{z}}$ and $\mathrm{J}_{\mathrm{x}}$ are not linearly independent.)

The procedure is as follows:

(i) Post-process the original code result (with conducting wall $\mathrm{BC}$ ) to obtain $\mathrm{J}_{\mathrm{x}}$ and $\mathrm{B}_{\mathrm{y}}$ on the surface

(ii) Solve Eqs. (3) - (6) with $\mathrm{z}_{\mathrm{sh}}=0$ using $\mathrm{J}_{\mathrm{x}}$ and $\mathrm{B}_{\mathrm{y}}$ as inputs to obtain the amplitudes of the four modes, $\mathrm{E}^{(\mathrm{m})}, \mathrm{m}=1,2,3,4$. Note that the polarization vectors of the modes are known from the eikonal solution so only the amplitudes are unknown and there are four equations for these four unknowns.

(iii) Solve Eqs. (3) - (6) with $z_{s h}$ specified as the desired sheath impedance, using as inputs the amplitudes of the two incoming modes from step (ii) (in general, one FW and one SW) . Solve for new values of $\mathrm{J}_{\mathrm{x}}$ and $\mathrm{B}_{\mathrm{y}}$ as well as new amplitudes of the two outgoing modes.

Finally for a self-consistent sheath impedance, the dependence $\mathrm{z}_{\mathrm{sh}}\left(\left|\Phi_{\mathrm{sh}}\right|\right)$ on $\Phi_{\mathrm{sh}}$ must be taken into account, i.e. the equation

$$
\Phi_{\mathrm{sh}}=-\mathrm{J}_{\mathrm{n}} \mathrm{z}_{\mathrm{sh}}\left(\left|\Phi_{\mathrm{sh}}\right|\right)
$$

must also be satisfied at each point on the surface. A root-finder for Eq. (7) may additionally be imposed on the procedure. In some cases, iteration may be sufficient, in which case we may add a final step

(iv) Calculate the new sheath potential from Eq. (7), and using the updated value of $\mathrm{z}_{\mathrm{sh}}$ for this sheath potential, repeat steps (iii) and (iv) until convergence.

Note that the $\mathrm{k}_{\mathrm{y}}$ in Eq. (4) assumes that the spatial variation of the envelope $\left|\Phi_{\text {sh }}\right|$ in Eq. (7) is slow compared with that of the phase; this could be improved by employing Fourier transforms in y on the whole term as discussed subsequently. Note that in some cases Eq. (7) [e.g. coupled to Eq. (18)] may have multiple roots so that a single solution with metallic BCs may result in more than one solution with sheath BCs. Further discussion of multiple roots is beyond the scope of this paper; the issues are independent of the post-processing method.

\section{Sample application}

As an example application, intended mainly as a first proof-of-principle test of the procedure, we now consider the case of a perpendicular sheath (i.e. one for which the magnetic field is perpendicular to the surface) in a slab geometry with constant plasma parameters. The geometry is illustrated in Fig. 1 and is the same as described in Ref. [10] for the 2D slab version of the rfSOL code.

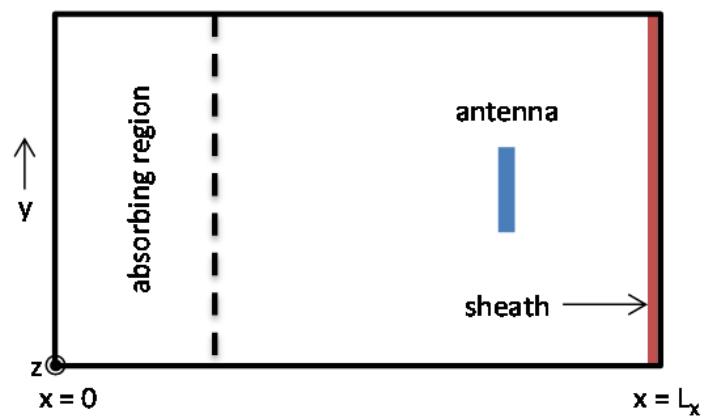

Fig. 1. Geometry of the sample application.

A sheet current antenna launches waves that propagate (or are evanescent) both to the left and right of it. The right propagating branch interacts with the sheath 
boundary condition of surface impedance $z_{s h}$ at $x=L_{x}$. In general there is reflection from the surface; this left going branch together with the one launched directly by the antenna are absorbed before they reach the left domain boundary at $\mathrm{x}=0$ creating an outgoing/ evanescent wave condition in this direction. The simulations are periodic in $\mathrm{y}$ and for the perpendicular incidence problem considered here the background magnetic field is $\mathbf{B}_{0}=\mathbf{e}_{\mathrm{x}} \mathrm{B}_{0 \mathrm{x}}$. With walls normal to $\mathbf{B}_{0}$ the FW does not contribute to RF sheath excitation and a SW analysis will suffice.

The rfSOL code was first run with conducting wall boundary conditions at $\mathrm{x}=\mathrm{L}_{\mathrm{x}}$ equivalent to $\mathrm{z}_{\mathrm{sh}}=0$. From this solution we extract the incoming wave amplitude (i.e. the wave incident on the $\mathrm{L}_{\mathrm{x}}$ boundary) as follows. The general solution for the RF wave in the electrostatic limit (to be generalized subsequently) is

$$
\begin{aligned}
& \mathbf{E}=-\mathbf{i k}_{\mathrm{sw}} \Phi^{(1)} \mathrm{e}^{\mathrm{ik} \mathrm{sw}, \mathrm{x}_{\mathrm{x}}\left(\mathrm{x}-\mathrm{L}_{\mathrm{x}}\right)} \\
& +\mathrm{i} \mathbf{k}_{\mathrm{sw}} \Phi^{(2)} \mathrm{e}^{-\mathrm{i} \mathrm{k}_{\mathrm{sw}, \mathrm{x}}\left(\mathrm{x}-\mathrm{L}_{\mathrm{x}}\right)}
\end{aligned}
$$

where $\Phi^{(1)}$ and $\Phi^{(2)}$ are the amplitudes of the electrostatic potential for the incoming and outgoing waves respectively. By convention $\mathrm{k}_{\mathrm{sw}, \mathrm{x}}$ is taken as the root with $\mathrm{k}_{\mathrm{sw}, \mathrm{x}}>0$. The total potential at the wall is

$$
\Phi=\Phi^{(1)}+\Phi^{(2)}
$$

which must be zero for the conducting wall (cw) case. Therefore at the wall

$$
\mathrm{E}_{\mathrm{X}}=-2 \mathrm{ik}_{\mathrm{sw}, \mathrm{x}} \Phi^{(1)}
$$

The total $E_{x}$ and $D_{x} \equiv i J_{x} / \omega$ at the wall are related by

$$
\varepsilon_{0} \varepsilon_{\|} \mathrm{E}_{\mathrm{x}}=\mathrm{D}_{\mathrm{x}}
$$

where $\varepsilon_{0}$ is the vacuum dielectric constant and $\varepsilon_{\|}$is the parallel (diagonal) component of the cold plasma dielectric tensor. Thus we have

$$
\Phi^{(1)}=\frac{\mathrm{iD}_{\mathrm{x}, \mathrm{cw}}}{2 \varepsilon_{0} \varepsilon_{\|} \mathrm{k}_{\mathrm{sw}, \mathrm{x}}}
$$

Although it is not necessary for the method, here for simplicity we make the assumption that the waves near the boundary can be treated in the local approximation $\mathrm{k}_{\mathrm{z}}$ $>\mathrm{k}_{\mathrm{y}}$ where $\mathrm{k}_{\mathrm{y}} \approx \mathrm{k}_{\perp}$ is a typical wavenumber in the $\mathrm{y}$ direction. (In general for $\mathrm{k}_{\mathrm{y}} \sim \mathrm{k}_{\mathrm{z}}$ each Fourier mode in $\mathrm{y}$ would be treated independently and summed up). The SW dispersion relation gives

$$
\mathrm{k}_{\mathrm{sw}, \mathrm{x}}^{2}=\mathrm{k}_{\|}^{2}=\frac{-\mathrm{k}_{\perp}^{2} \varepsilon_{\perp}}{\varepsilon_{\|}}
$$

completing the determination of $\Phi^{(1)}$.

Next we turn to the solution for a specified value of $\mathrm{z}_{\mathrm{sh}}$. The basic assumption is that $\Phi^{(1)}$ remains unchanged from the value in Eq. (12). Thus we solve the system

$$
\begin{gathered}
\varepsilon_{0} \varepsilon_{\|} \mathrm{E}_{\mathrm{X}}=\mathrm{D}_{\mathrm{x}} \\
\Phi^{(1)}+\Phi^{(2)} \equiv \Phi_{\mathrm{sh}}=-\mathrm{i} \omega \mathrm{D}_{\mathrm{X}} \mathrm{z}_{\mathrm{sh}}
\end{gathered}
$$

now regarding $\Phi^{(1)}$ as known and $\Phi^{(2)}$ and $\mathrm{D}_{\mathrm{x}}$ as the unknowns. $E_{x}$ in Eq. (14) is expressed in terms of $\Phi^{(1)}$ and $\Phi^{(2)}$ from the expansion given in Eq. (8). After a small amount of algebra, the result is

$$
\begin{gathered}
\mathrm{D}_{\mathrm{x}}=\frac{\mathrm{D}_{\mathrm{x}, \mathrm{cw}}}{1-\omega \varepsilon_{0} \varepsilon_{\|} \mathrm{k}_{\mathrm{sw}, \mathrm{x}} \mathrm{z}_{\mathrm{sh}}} \\
\Phi^{(2)}=-\frac{\mathrm{iD} \mathrm{x}_{\mathrm{x}, \mathrm{cw}}}{2 \varepsilon_{0} \varepsilon_{\|} \mathrm{k}_{\mathrm{sw}, \mathrm{x}}}\left(\frac{1+\omega \varepsilon_{0} \varepsilon_{\|} \mathrm{k}_{\mathrm{sw}, \mathrm{x}} \mathrm{z}_{\mathrm{sh}}}{1-\omega \varepsilon_{0} \varepsilon_{\|} \mathrm{k}_{\mathrm{sw}, \mathrm{x}} \mathrm{z}_{\mathrm{sh}}}\right)
\end{gathered}
$$

The RF sheath voltage $\Phi_{\text {sh }}=\Phi^{(1)}+\Phi^{(2)}$ is therefore

$$
\Phi_{\mathrm{sh}}=\frac{-\mathrm{i} \omega \mathrm{D}_{\mathrm{x}, \mathrm{cw}} \mathrm{z}_{\mathrm{sh}}}{1-\omega \varepsilon_{0} \varepsilon_{\|} \mathrm{k}_{\mathrm{sw}, \mathrm{x}} \mathrm{z}_{\mathrm{sh}}}
$$

If $z_{s h}$ is very small then it is clear that the conducting wall solution is recovered. In the opposite limit of large $\mathrm{z}_{\mathrm{sh}}$ (the quasi-insulating limit) the current $\mathrm{J}_{\mathrm{x}}$ into the wall, proportional to $\mathrm{D}_{\mathrm{x}}$, goes to zero and the sheath voltage is $\Phi_{\mathrm{sh}}=\mathrm{iD}_{\mathrm{x}, \mathrm{cw}} / \varepsilon_{0} \varepsilon_{\|} \mathrm{k}_{\mathrm{sw}, \mathrm{x}}$. In intermediate cases, if $\mathrm{z}_{\mathrm{sh}}$ and $\mathrm{k}_{\mathrm{sw}, \mathrm{x}}$ are nearly pure imaginary (possible in the capacitive sheath limit with evanescent SWs), sheathplasma resonance occurs and can result in large values of $\Phi_{\text {sh}}$.

It is not difficult to show that in the electromagnetic (EM) case the SW dispersion relation, Eq. (13) is replaced by

$$
\mathrm{n}_{\perp}^{2} \varepsilon_{\perp}+\mathrm{n}_{\mathrm{sw}, \mathrm{x}}^{2} \varepsilon_{\|}=\varepsilon_{\perp} \varepsilon_{\|}
$$

and the final results in Eqs. (16) - (18) only require the replacement of $\mathrm{k}_{\mathrm{sw}, \mathrm{x}}$ with $\mathrm{q}_{\mathrm{sw}, \mathrm{x}}$ given by

$$
\mathrm{q}_{\mathrm{sw}, \mathrm{x}}=\mathrm{k}_{\mathrm{sw}, \mathrm{x}} \frac{\mathrm{n}_{\perp}^{2}}{\mathrm{n}_{\perp}^{2}-\varepsilon_{\|}}
$$

where $\mathbf{n}=\mathbf{k c} / \omega$.

For a test to compare with rfSOL we considered an evanescent EM SW case. The excitation of the FW can be neglected. The main parameters of interest here are $\mathrm{B}_{0 \mathrm{x}}=1 \mathrm{~T}$, density $\mathrm{n}_{\mathrm{e}}=2 \times 10^{18} \mathrm{~m}^{-3}, \mathrm{~T}_{\mathrm{e}}=15 \mathrm{eV}, \mathrm{RF}$ wave frequency $\mathrm{f}=\omega /(2 \pi)=80 \mathrm{MHz}$, antenna-sheath distance $=0.2 \mathrm{~m}$, and the specified wavenumber $\mathrm{k}_{\mathrm{z}}=$ $160 \mathrm{~m}^{-1}$. The dominant mode launched by the antenna is $\mathrm{k}_{\mathrm{y}}=120 \mathrm{~m}^{-1}$ and is taken as an input parameter here. The antenna current is $1.1 \mathrm{kA} / \mathrm{m}$ which results in a maximum (in y) sheath voltage of about $130 \mathrm{~V}$. Other parameters, of less interest for present purposes, are given in Ref. [10]; see Fig. 11(a).

For these parameters, $\omega / \omega_{\text {pi }}=0.38$ resulting in a complex impedance with significant real part, and $\left|\mathrm{n}_{\perp}^{2} / \varepsilon_{\|}\right|=0.57$ implying important EM effects. Furthermore, it turns out that $\mathrm{z}_{\mathrm{sh}}$ is not so large as to put 
the final result in the quasi-insulating limit; thus, the results are still sensitive to the value of $z_{\text {sh }}$. This case therefore provides a robust test of the method. Iteration, updating the nonlinear dependence [9] of $\mathrm{z}_{\mathrm{sh}}\left(\left|\Phi_{\mathrm{sh}}\right|\right)$, was carried out using the same fits as in rfSOL [10] to obtain a self-consistent solution at each value of y. Starting from an initial constant value of either essentially infinite $\mathrm{z}_{\mathrm{sh}}$ or from $\mathrm{z}_{\mathrm{sh}}=0$ the solution converges rapidly.

As can be seen from Fig. 2, the comparison between the post-processing solution and the rfSOL result is quite good. The main differences are likely the result of employing local theory for the post-processing, which begins to lose accuracy when only the dominant harmonic in $\mathrm{k}_{\mathrm{y}}$ is retained, as done here. As explained previously, local theory is not essential to the method, but is convenient for the present test.

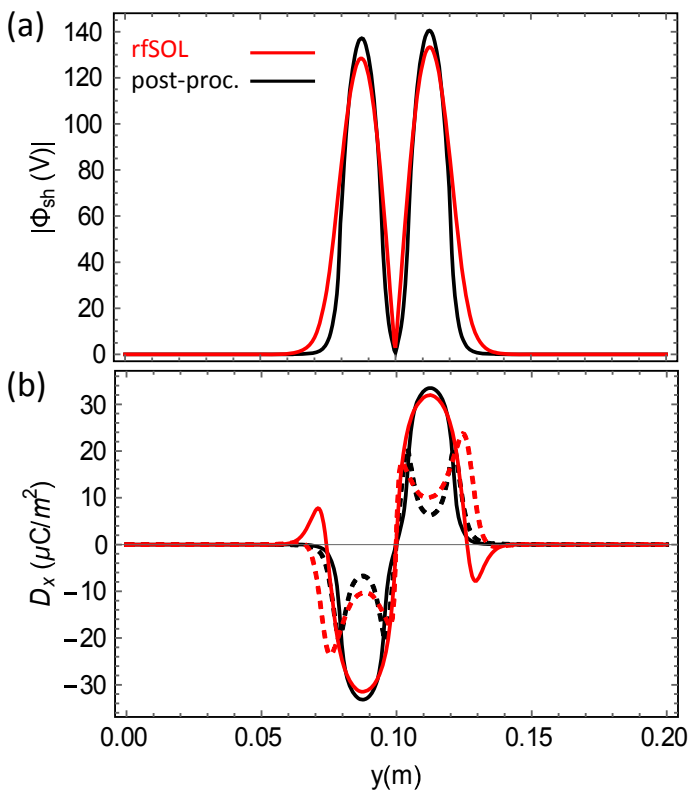

Fig. 2. Comparison of the post-processing solution (black) with the numerical solution obtained from rfSOL (red) for the electromagnetic test case: (a) the magnitude of the rf sheath voltage; (b) real (solid) and imaginary (dashed) parts of $\mathrm{D}_{\mathrm{x}}$.

\section{Discussion and conclusions}

The main assumption of the method is that the amplitude of the incoming waves is not changed by the $\mathrm{BC}$. In the example this is assured by the fact that the SW is evanescent on a scale shorter than the distance between the walls and (in general if necessary) the absorbing layer. In addition, the present analysis has also assumed locally constant plasma parameters which enable a local solution for $\mathrm{k}_{\mathrm{x}}$ given specified values of $\mathrm{k}_{\mathrm{y}}$ and $\mathrm{k}_{\mathrm{z}}$. Here $\mathrm{k}_{\mathrm{y}}$ and $\mathrm{k}_{\mathrm{z}}$ may be obtained from the Fourier transform of the normal current $\sim \mathrm{D}_{\mathrm{x}}$ on the surface. For $2 \mathrm{D}$ codes $\mathrm{k}_{\mathrm{z}}$ may be calculated from the mode number in the toroidal direction. If there is no dominant $\mathrm{k}_{\mathrm{y}}$, then the procedure may be applied to each $\mathrm{k}_{\mathrm{y}}$ in the spectrum. The value of $\mathrm{k}_{\mathrm{x}}$ for each mode i.e. propagation direction of $\mathrm{SW}$ and
FW $(\mathrm{m}=1,2,3,4)$ is given from the local dispersion relation for the chosen $\mathrm{k}_{\mathrm{y}}$ and $\mathrm{k}_{\mathrm{z}}$. In the illustration of the method in Eqs. (3) - (6), the tangential $\mathbf{k}$ is assumed to be equal for FW and $\mathrm{SW}$, but this could be generalized. The use of local wavenumbers $\mathrm{k}_{\mathrm{x}}, \mathrm{k}_{\mathrm{y}}$ and $\mathrm{k}_{\mathrm{z}}$ to describe the fields near the surface is expected to be rigorous when the surface is locally flat on the scale of the waves and the plasma parameters are constant on that scale. Note that in the case of $\mathrm{FW} \rightarrow \mathrm{SW}$ conversion due to wall shape (e.g. a wall "bump") [8] the conducting wall solution for $\mathrm{D}_{\mathrm{x}}$ will contain $\mathrm{k}_{\mathrm{y}}$ values characteristic of the bump scale.

We have shown that a sheath post-processing method can be used to transform a solution obtained with a conducting wall $\mathrm{BC}$ to one with a general impedance sheath BC. Used in this way, the method allows legacy rf codes (with conducting wall BCs) to obtain approximate rf sheath properties by post-processing. In fact, the same procedure can be used to transform between any two different cases of an impedance boundary condition (for example from an insulating sheath solution where $\mathrm{z}_{\mathrm{sh}} \rightarrow \infty$ to a finite $\mathrm{z}_{\mathrm{sh}}$ solution).

In the case of a locally curved surface, or if the density or magnetic field vary significantly with respect to the SW wavelength near the wall, then the eikonal ansatz could introduce significant approximations. Further work will be required to assess the accuracy of the method for practical applications in such cases.

This material is based upon work supported by the U.S. Department of Energy Office of Science, Office of Fusion Energy Sciences under Award Numbers DE-FG02-97ER54392 and DE-FC02-05ER54823. The digital data for this paper can be found at https://doi.org/10.5281/zenodo.579548.

\section{References}

1. R. J. Perkins, J. C. Hosea, M. A. Jaworski, J.-W. Ahn et al., Phys. Plasmas 22, 042506 (2015)

2. V. Bobkov, D. Aguiam, R. Bilato, S. Brezinsek et al., Plasma Phys. Control. Fusion 59, 014022 (2017)

3. S. J. Wukitch, M. L. Garrett, R. Ochoukov, J. L. Terry et al. Phys. Plasmas 20, 056117 (2013)

4. E. F. Jaeger, L. A. Berry, J. S. Tolliver, D.B. Batchelor, Phys. Plasmas 2, 2597 (1995)

5. D. A. D'Ippolito and J. R. Myra, Phys. Plasmas 13, 102508 (2006)

6. L. Colas, J. Jacquot, S. Heuraux, E. Faudot, K. Crombé et al., Phys. Plasmas 19, 092505 (2012)

7. D. Van Eester, K. Crombé and V. Kyrytsya, Plasma Phys. Control. Fusion 55, 055001 (2013)

8. H. Kohno, J. R. Myra, and D. A. D'Ippolito, Phys. Plasmas 22, 072504 (2015); and Phys. Plasmas 23, 089901 (2016)

9. J. R. Myra and D. A. D’Ippolito, Phys. Plasmas 22, 062507 (2015)

10. H. Kohno and J. R. Myra, in press, Comp. Phys. Comm. (2017) 